(C) 1998 International Press

Adv. Theor. Math. Phys. 2 (1998) 571-591

\title{
The Higgs Branch of Impurity Theories
}

\author{
Anton Kapustin ${ }^{1}$, Savdeep Sethi ${ }^{2}$ \\ School of Natural Sciences \\ Institute for Advanced Study \\ Princeton, NJ 08540, U. S. A.
}

\begin{abstract}
We consider supersymmetric gauge theories with impurities in various dimensions. These systems arise in the study of intersecting branes. Unlike conventional gauge theories, the Higgs branch of an impurity theory can have compact directions. For models with eight supercharges, the Higgs branch is a hyperKähler manifold given by the moduli space of solutions of certain differential equations. These equations are the dimensional reductions of self-duality equations with boundary conditions determined by the impurities. They can also be interpreted as Nahm transforms of self-duality equations on toroidally compactified spaces. We discuss the application of our results to the light-cone formulation of Yang-Mills theories and to the solution of certain $\mathrm{N}=2$ $\mathrm{d}=4$ gauge theories.
\end{abstract}

\section{Introduction}

Intersecting brane configurations are important in both string theory and matrix theory [1]. When the branes involved are Dirichlet, we should be able to describe the dynamics of the intersecting brane configuration in terms of a gauge theory with some degrees of freedom localized at the intersection.

e-print archive: http://xxx.lanl.gov/abs/hep-th/9804027

${ }^{1}$ E-mail: kapustin@sns.ias.edu

${ }^{2}$ E-mail: sethi@sns.ias.edu 
These degrees of freedom come from strings stretching between the intersecting branes. We will call theories with localized degrees of freedom 'impurity theories' $[2,3]$.

The aim of this paper is to analyze the impurity theories that arise from the intersection of partially compactified D-branes. For example, the theory describing the dynamics of $N$ D0-branes in the presence of $k$ D4-branes wrapped on a circle should have two branches. On the Higgs branch, the fundamental hypermultiplets coming from the D0-D4 strings have expectation values. The $\mathrm{D} 0$-branes then cannot leave the $\mathrm{D} 4$-branes since the moduli parametrizing motion away from the D4-branes are massive. The D0-branes essentially become instantons living in the D4-branes. The Higgs branch of this impurity theory must have compact directions since it should be describing the moduli space of instantons on $\mathbb{R}^{3} \times S^{1}$. On the Coulomb branch, we can give expectation values to the moduli parametrizing motion away from the D4-branes. On this branch, the fundamental hypermultiplets are massive. Similarly, when the D4-branes are wrapped on a more general space, the Higgs branch should describe the instantons on this space and therefore can have compact directions.

Our goal is to show how compact moduli spaces appear in impurity theories. The models we consider have eight supercharges and non-chiral impurities. In these cases, the Higgs branch is hyperKähler and not renormalized by quantum corrections. In the following section, we derive the Lagrangian for particular impurity theories and analyze the Higgs branch. In section three, we explain how the Higgs branch is related to the Nahm transform of the self-duality equations on $\mathbb{R}^{3} \times S^{1}$ and $\mathbb{R}^{2} \times T^{2}$. We conclude by explaining how our results are applicable to matrix formulations of Yang-Mills theories, and to the solution of certain $\mathrm{N}=2 \mathrm{~d}=4$ theories.

It would be interesting to extend this analysis to impurity theories with fewer supersymmetries and to theories with localized chiral matter. Such systems arise, for example, in the study of type I D1-branes in the presence of D5-branes [4] wrapped on tori. Related results have been obtained by $[5,6]$.

\section{Supersymmetric Vacua of Impurity Systems}

\subsection{The Supersymmetric Lagrangian}

The primary example that we will study is the system of $N$ D0-branes probing $k \mathrm{D} 4$-branes wrapped on tori. To derive the impurity Lagrangian, we use the approach pioneered in $[7,8]$, and extended to the impurity case in $[2,3]$. Let us start with the case of four-branes on $\mathbb{R}^{4}$. The theory on the D0-branes is then a $U(N)$ quantum mechanics with $k$ fundamental hypermultiplets and 
one adjoint hypermultiplet. For simplicity, we will take D4-branes to be coincident. Separating the four-branes amounts to turning on bare masses for the fundamental hypermultiplets.

The field content can be obtained by dimensionally reducing an $N=2$ gauge theory in $d=4$. Our conventions are similar to those in [9]. The global symmetry group is $\operatorname{Spin}(5) \times S U(2)_{R} \times S U(2)$, and a vector multiplet contains five scalars which we call $Y^{i}, i=1, \ldots, 5$, and a pair of symplectic Majorana fermions $\lambda^{\alpha}, \alpha=1,2$ transforming as a doublet under the $S U(2)_{R}$ symmetry (we are using a 'four-dimensional' language to describe the fermions). The adjoint hypermultiplet has two complex scalars $H^{\alpha}$, $\alpha=1,2$ forming a doublet under $S U(2)_{R}$ and a doublet with respect to the other $S U(2)$. It also contains a Dirac fermion $\psi$. Lastly, the $k$ fundamental hypermultiplets each contain two complex scalars $Q^{\alpha p}$, where $\alpha=1,2$ are the $S U(2)_{R}$ indices and $p=1, \ldots, k$ are the flavor indices, together with associated fermions. The adjoint hypermultiplet encodes degrees of freedom from the $0-0$ strings. Its four real scalars parametrize the motion of the D0-branes along the world-volume of the D4-branes. We will write all real adjoint fields as anti-Hermitian matrices. The bosonic part of the Lagrangian is a sum of three terms,

$$
L=L_{1}+L_{2}+L_{3} .
$$

The first contains the vector multiplet kinetic terms,

$$
L_{1}=\int d t\left(\frac{1}{2}\left|D_{0} Y^{i}\right|^{2}-\frac{1}{2} \sum_{i<j}\left|\left[Y^{i}, Y^{j}\right]\right|^{2}\right) .
$$

The covariant derivative is the usual one,

$$
D_{0}=\partial_{t}-A_{0} .
$$

The second piece contains the kinetic terms of the adjoint hypermultiplet,

$$
L_{2}=\int d t\left(\left|D_{0} H^{\alpha}\right|^{2}-\sum\left|\left[Y^{i}, H^{\alpha}\right]\right|^{2}\right),
$$

The final term contains the fundamental hypermultiplets and $\mathcal{D}$-terms,

$$
\begin{aligned}
L_{3}=\int d t\left(\left|D_{0} Q^{\alpha}\right|^{2}-\sum \mid\right. & \left.Y^{i} Q^{\alpha}\right|^{2}+\frac{1}{2}|\mathcal{D}|^{2} \\
& \left.+\operatorname{Tr} i \mathcal{D}_{\beta}^{\alpha}\left(\left[H_{\alpha}, H^{\dagger \beta}\right]+Q_{\alpha} \otimes Q^{\dagger \beta}\right)\right),
\end{aligned}
$$

where flavor indices have been suppressed. In eq. (2.3),

$$
\mathcal{D}_{\beta}^{\alpha}=\mathcal{D}^{a}\left(\sigma^{a}\right)_{\beta}^{\alpha},
$$


where $\mathcal{D}^{a} a=1,2,3$ is a triplet of auxiliary fields in the adjoint. The $S U(2)_{R}$ indices are moved up and down using $\epsilon_{\alpha \beta}$ so that $\mathcal{D}^{\alpha \beta}$ is symmetric. The tensor product refers to the $U(N)$ (color) indices.

Let us pick $X^{1}=\sqrt{2} \operatorname{Re} H_{1}$ as the longitudinal direction which we wish to compactify. We then take the system and all its translates along $X^{1}$. This describes an array of an infinite number of D0-branes. The gauge group is now infinite-dimensional and we quotient by the symmetry group generated by translations along $X^{1}$ by $2 \pi i R_{1}[7,8]$. Recall that $X^{1}$ is anti-Hermitian. More explicitly, we impose the constraints:

$$
\begin{aligned}
& Y_{n m}^{i}=Y_{(n-1)(m-1)}^{i}, \\
& H_{n m}^{\prime}=H_{(n-1)(m-1)}^{\prime}, \\
& \mathcal{D}_{n m}=\mathcal{D}_{(n-1)(m-1)}, \\
& X_{n m}^{1}=X_{(n-1)(m-1)}^{1} \quad n \neq m, \\
& X_{n n}^{1}=X_{(n-1)(n-1)}^{1}+2 \pi i R_{1},
\end{aligned}
$$

where $H^{\prime}$ excludes $X^{1}$ and the subscripts $n, m$ label the translate. Note that each component, say $Y_{n m}$, is still an $N \times N$ matrix transforming in the adjoint representation of our original $U(N)$ gauge group. As usual, it proves convenient to perform a Fourier transform on all the adjoint fields, e.g.

$$
Y^{i}\left(x^{1}\right)=\sum_{n} Y_{n 0}^{i} e^{2 \pi i n R_{1} x_{1}}
$$

This promotes $Y, H^{\prime}, \mathcal{D}$ to fields living on a circle $\widehat{S}^{1}$ of radius $\widehat{R}_{1}=1 / 2 \pi R_{1}$. As for $X^{1}$, its Fourier transform is a differential operator rather than a function:

$$
X^{1}\left(x^{1}\right)=\partial_{1}-A_{1}\left(x_{1}\right)
$$

The fundamental hypermultiplets coming from the D0-D4 strings are treated differently since the D4-branes are longitudinal. The $p^{\text {th }}$ hypermultiplets $Q^{p}$ obeys,

$$
Q_{n}^{p}=e^{-2 \pi i R_{1} n s_{p}} Q_{0}^{p}
$$

where $s_{1}, \ldots, s_{k}$ are real numbers. They parametrize the Wilson lines for the $U(k)$ gauge theory on the D4-branes. The fundamental hypermultiplets are therefore not promoted to fields on $\widehat{S}^{1}$. We will see in a moment that the $p^{\text {th }}$ hypermultiplet is in fact 'localized' at $x^{1}=s_{p}$.

After substituting the expressions for $Y$ and $H$ in terms of their Fourier 
transforms into eq. (2.1) and eq. (2.2), we obtain:

$$
\begin{gathered}
L_{1}+L_{2}=R_{1} \int d t d x_{1}\left[\frac{1}{2}\left|F_{01}\right|^{2}+\frac{1}{2}\left|D_{0} Y^{i}\right|^{2}-\frac{1}{2}\left|D_{1} Y^{i}\right|^{2}-\frac{1}{2} \sum_{i<j}\left|\left[Y^{i}, Y^{j}\right]\right|^{2}\right. \\
\left.+\left|D_{0} \operatorname{Im} H_{1}\right|^{2}+\left|D_{0} H_{2}\right|^{2}-\sum_{i}\left(\left|\left[Y^{i}, H_{2}\right]\right|^{2}+\left|\left[Y^{i}, \operatorname{Im} H_{1}\right]\right|^{2}\right)\right] \cdot \quad(2.8)
\end{gathered}
$$

Here $F_{01}=-\left[D_{0}, D_{1}\right]$. The more interesting terms in the Lagrangian involve the couplings to $Q$. The terms in $L_{3}$ become,

$$
\begin{gathered}
L_{3}=R_{1} \int d x_{1} d t\left[\sum_{p=1}^{k}\left(\frac{1}{R_{1}} \delta\left(x_{1}-s_{p}\right)\left(\left|D_{0} Q^{\alpha p}\right|^{2}-\sum\left|Y^{i}\left(x_{1}\right) Q^{\alpha p}\right|^{2}\right)\right)\right. \\
\left.+\frac{1}{2}|\mathcal{D}|^{2}+\operatorname{Tr} i \mathcal{D}_{\beta}^{\alpha}\left(\left[H_{\alpha}, H^{\dagger \beta}\right]+\frac{1}{R_{1}} \sum_{p=1}^{k} \delta\left(x_{1}-s_{p}\right) Q_{\alpha}^{p} \otimes Q^{\dagger p \beta}\right)\right], \quad
\end{gathered}
$$

where it is understood that Re $H_{1}$ is replaced by $\frac{1}{\sqrt{2}}\left(\partial_{1}-A_{1}\right)$, and the $\delta$ function on $\widehat{S}^{1}$ is defined so that $\oint d x^{1} \delta\left(x_{1}\right)=1$. After integrating out the auxiliary field $\mathcal{D}$, the resulting Lagrangian will contain terms proportional to $\delta(0)$ which may seem problematic. However, this should not be very surprising in theories with boundary interactions. For example, $\mathrm{M}$ theory on $S^{1} / \mathbb{Z}_{2}$ which is relevant to the strong-coupling limit of the $E_{8} \times E_{8}$ heterotic string, has similar divergences appearing in the Lagrangian [10]. As we shall see, the $\delta$-function terms in $\mathcal{D}$-terms are crucial if we are to obtain a Higgs branch with the properties that we expect on physical grounds.

\section{$2.2 \quad \mathbb{R}^{3} \times S^{1}$}

Let us first consider the case of $N$ D0-branes in the presence of $k \mathrm{D} 4$-branes wrapped on a circle of radius $R_{1}$. On the Higgs branch, we set $Y=0$ but allow the $Q$ hypermultiplets to have non-zero expectation values. With this choice, the supersymmetric variation of the 'squarks', the superpartners of $Q$, automatically vanishes. To ensure that we have a supersymmetric vacuum, we must force the supersymmetric variation of the gluinos to vanish as well. This reduces to the condition that the $\mathcal{D}$-terms vanish. Note, however, that this condition is now a differential equation because $\operatorname{Re} H_{1}$ is a differential operator. The vacuum is therefore not spatially homogeneous. To write the $\mathcal{D}$-flatness condition in a more familiar form, we denote:

$$
\begin{aligned}
& T_{0}=-A_{1}, \quad T_{1}=-\sqrt{2} \operatorname{Im} H_{1}, \quad T_{2}+i T_{3}=-\sqrt{2} H_{2}, \\
& Q=Q_{1}, \quad \tilde{Q}=\left(Q_{2}\right)^{\dagger} .
\end{aligned}
$$


We can then rewrite the equations to get,

$$
\begin{aligned}
& \frac{d T_{1}}{d x_{1}}+\left[T_{0}, T_{1}\right]+\left[T_{2}, T_{3}\right]=-\frac{i}{R_{1}} \sum_{p=1}^{k} \delta\left(x_{1}-s_{p}\right)\left(Q^{p} \otimes Q^{\dagger p}-\tilde{Q}^{\dagger p} \otimes \tilde{Q}^{p}\right) \\
& \frac{d T_{2}}{d x_{1}}+\left[T_{0}, T_{2}\right]+\left[T_{3}, T_{1}\right]=-\frac{i}{R_{1}} \sum_{p=1}^{k} \delta\left(x_{1}-s_{p}\right)\left(-i Q^{p} \otimes \tilde{Q}^{p}+i \tilde{Q}^{\dagger p} \otimes Q^{\dagger p}\right) \\
& \frac{d T_{3}}{d x_{1}}+\left[T_{0}, T_{3}\right]+\left[T_{1}, T_{2}\right]=-\frac{i}{R_{1}} \sum_{p=1}^{k} \delta\left(x_{1}-s_{p}\right)\left(Q^{p} \otimes \tilde{Q}^{p}+\tilde{Q}^{\dagger p} \otimes Q^{\dagger p}\right) .
\end{aligned}
$$

These equations without the right-hand side source terms are known as Nahm equations. They play an important role in the construction of selfdual monopoles and calorons. ${ }^{3}$ We will discuss these equations in more detail in section three.

If the VEVs of the $Q$ and $\tilde{Q}$ hypermultiplets are non-zero, eqs. (2.11) force $T_{i}, i=1,2,3$, to have step-like discontinuities at the points $s_{1}, \ldots, s_{k}$. Let us see how this picture is interpreted in terms of the D-branes. After T-duality along the $S^{1}$, we are studying a system of $N$ D1-branes wrapped on $\widehat{S}^{1}$ with $k$ D3-branes located at $k$ points $s_{1}, \ldots, s_{k}$ on $\widehat{S}^{1}$. The VEVs of the $T_{i}$ fields parametrize the positions of the D1-branes in the directions $x^{2}, x^{3}, x^{4}$ parallel to the D3-branes. The discontinuity in $T_{i}$ at $x_{1}=s_{p}$ is now interpreted as D1-strings breaking at the $p^{\text {th }}$ D3-brane, as pictured in Figure 1. This breaking prevents the D1-strings from moving off in the directions perpendicular to the D3-branes. This means that fields in the vector multiplet are massive.

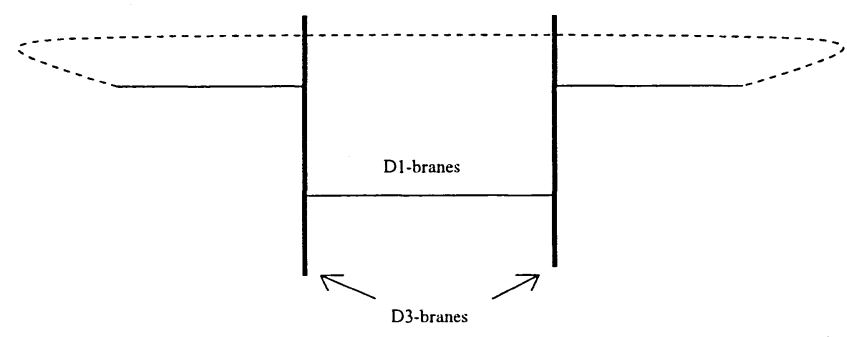

Figure 1: Jumps in $T_{i}$.

Naturally, we should consider the solutions of the $\mathcal{D}$-flatness equations modulo $U(N)$ gauge transformations. The moduli space of solutions to the $\mathcal{D}$-term equations should then coincide with the moduli space of $N U(k)$ instantons on $\mathbb{R}^{3} \times S^{1}$ with the fixed Wilson lines. We will give another explanation of this in section three.

\footnotetext{
${ }^{3}$ Otherwise known as instantons on $\mathbb{R}^{3} \times S^{1}$.
} 


\section{$2.3 \quad \mathbb{R}^{2} \times T^{2}$}

Let us compactify another direction along the D4-branes, say $X^{2}=\sqrt{2} \operatorname{Im} H_{1}$, on a circle of radius $R_{2}$. The procedure described before goes through in the same way. The adjoint degrees of freedom become fields on the dual torus $\widehat{T}^{2} ;$ in particular,

$$
X^{2} \rightarrow \partial_{2}-A_{2}
$$

The fundamental degrees of freedom are localized at points of $\widehat{T}^{2} z_{1}, \ldots, z_{k}$ which encode the $U(k)$ Wilson lines on $T^{2}$. Let us define $A_{\bar{z}}=\frac{1}{2}\left(A_{1}+\right.$ $\left.i A_{2}\right), \Phi=\frac{1}{\sqrt{2}} H^{\dagger 2}$. Then the Higgs branch is described by the moduli space of solutions of the equations,

$$
\begin{aligned}
& F_{z \bar{z}}-\left[\Phi, \Phi^{\dagger}\right]=\frac{1}{2 R_{1} R_{2}} \sum_{p=1}^{k} \delta^{2}\left(z-z_{p}\right)\left(Q^{p} \otimes Q^{\dagger p}-\tilde{Q}^{\dagger p} \otimes \tilde{Q}^{p}\right) \\
& \bar{D} \Phi=-\frac{1}{2 R_{1} R_{2}} \sum_{p=1}^{k} \delta^{2}\left(z-z_{p}\right) Q^{p} \otimes \tilde{Q}^{p}
\end{aligned}
$$

Here $F_{z \bar{z}}=\partial A_{\bar{z}}-\bar{\partial} A_{z}-\left[A_{z}, A_{\bar{z}}\right]$ and $\bar{D}=\bar{\partial}-A_{\bar{z}}$.

These equations are Hitchin equations on the dual torus $\widehat{T}^{2}$ with impurities localized at points of $\widehat{T}^{2}$. Recalling that these equations describe $N$ D0-branes stuck to $k$ D4-branes wrapped around $\mathbb{R}^{2} \times T^{2}$, we expect that the moduli space of solutions of eq. (2.13) coincides with the moduli space of $N U(k)$ instantons on $\mathbb{R}^{2} \times T^{2}$ with prescribed Wilson lines.

If we compactify more than two directions, we run into a problem. The $\mathcal{D}$-flatness conditions will then involve $\delta^{3}(x)$ or $\delta^{4}(x)$ terms for $T^{3}$ or $T^{4}$ compactifications, respectively. These seem to be too singular source terms for the equations to admit any solutions with non-zero $Q$ expectation values. There is a physical way of seeing why these cases might be problematic. Consider the case of D4-branes wrapped around $T^{3}$. After T-dualizing D4branes on $T^{3}$, we obtain impurity D1-branes while our 'probe' D0-branes become D3-branes wrapped on $\widehat{T}^{3}$. We want to be able to localize the position of the impurities at points on $\widehat{T}^{3}$. This means that we want to give expectation values to scalars in the 1+1-dimensional theory on the D1branes. However, the theory on D1-branes does not have a moduli space, so we can no longer regard the position of the D1-branes as fixed. Our attempt to do so leads to equations for the Higgs branch with no nontrivial solutions. The same problem occurs for $T^{4}$ compactifications. To deal with these cases, we should be doing quantum mechanics on the impurity world-volume. 


\subsection{Fayet-Iliopoulos Parameters}

Since the gauge group of our impurity theories is $U(N)$, it is possible to modify the $\mathcal{D}$-flatness conditions by Fayet-Iliopoulos (FI) terms. More precisely, the gauge group is the group of maps from $\widehat{S}^{1}$ or $\widehat{T}^{2}$ to $U(N)$, so FI terms can be functions on $\widehat{S}^{1}$ or $\widehat{T}^{2}$. In the presence of FI terms the $\mathcal{D}$-flatness conditions are modified to

$$
\mathcal{D}_{i}=\xi_{i}
$$

where both $\mathcal{D}$ and $\xi$ are functions on $\widehat{S}^{1}$ or $\widehat{T}^{2}$. Naively, this seems to introduce a continuum of deformation parameters. However, it is easy to see that in fact only the average of $\xi$ over $\widehat{S}^{1}$ or $\widehat{T}^{2}$ matters. For example, in the case considered in section 2.2, a change of variables $T_{i}\left(x^{1}\right) \rightarrow T_{i}\left(x^{1}\right)+h_{i}\left(x^{1}\right)$ shifts $\xi_{i}$ by $d h_{i} / d x^{1}$. Since such a change of variables leaves the moduli space unchanged, we see that the only 'gauge-invariant' information is contained in $\oint d x^{1} \xi_{i}$. Similarly, in the case considered in section 2.3 the only invariant is $\int d^{2} z \xi_{i}(z, \bar{z})$.

Recall that in the case of instantons on $\mathbb{R}^{4}$ the modification of the ADHM equations by FI terms corresponds to a $B$-field flux [11]. This FI deformation has an interpretation in terms of non-commutative geometry [12] as instantons on a noncommutative generalization of $\mathbb{R}^{4}[13]$. As explained in the next section, Nahm and Hitchin equations with impurities are nothing but the Nahm transform of instantons on $\mathbb{R}^{3} \times S^{1}$ and $\mathbb{R}^{2} \times T^{2}$, respectively. The FI terms should again correspond to a background B-field flux. These deformed moduli spaces should therefore be interpreted as describing instantons on a non-commutative $\mathbb{R}^{3} \times S^{1}$ and $\mathbb{R}^{2} \times T^{2}$. That there is a single three-component FI parameter corresponds to the fact that there are three closed self-dual 2-forms on $\mathbb{R}^{3} \times S^{1}$ and $\mathbb{R}^{2} \times T^{2}$.

\subsection{More General Gauge Groups}

There is no reason why the preceeding analysis cannot be extended to more general groups. Most straightforward are the two cases that naturally arise in string theory with orientifolds. Instead of starting with a system of just D4-branes and D0-branes, we can place either an $\mathrm{O}^{-}$or $\mathrm{O}^{+}$orientifold plane parallel to the $\mathrm{D} 4$-branes without breaking more supersymmetry.

In the former case, we have either an even or an odd number of D4-branes. The D4-branes have an $S O(k)$ gauge symmetry while the D0-branes have an $S p(N)$ gauge group. There are $k$ half-hypermultiplets in the fundamental and a hypermultiplet in the antisymmetric representation. In the $\mathrm{O}^{+}$case, we can only have an even number of D4-branes giving an $S p(k)$ gauge symmetry. In this case, the D0-branes have an $S O(N)$ gauge symmetry with $k / 2$ hypermultiplets in the vector representation and a hypermultiplet in the symmetric representation. 
After compactifying a longitudinal direction, we will end up with a theory of $N$ D-strings with two orientifold 3-planes and $k \mathrm{D} 3$-branes. Away from the orientifold points, the gauge group on the D-strings is still $U(N)$. The only effect of the orientifold projection is to relate the matrices $T_{0}\left(x_{1}\right), T_{i}\left(x_{1}\right)$, as well as the impurity degrees of freedom, at points on the circle related by $x^{1} \rightarrow-x^{1}$. See $[14,15]$ for the analogous discussion in the case of monopoles. Similarly, in the case of compactification on $T^{2}$ we obtain the same equations (Hitchin equations) on $\widehat{T}^{2}$, with an additional identification under $z \rightarrow-z$. It would be interesting to investigate the corresponding moduli spaces in more detail for both $S^{1}$ and $T^{2}$.

\section{The Reduction of Self-duality Equations}

\subsection{HyperKähler Quotients}

In a conventional gauge theory with gauge group $G$, the Higgs branch is determined by solving the algebraic equation of $\mathcal{D}$-flatness,

$$
\mathcal{D}=0 .
$$

The moduli space of solutions has no compact directions for these models. It has a natural hyperKähler metric because the equation $\mathcal{D}=0$ defines a hyperKähler moment map for the group of gauge transformations $G$. Let us recall how this comes about. A hyperKähler manifold is a Riemannian manifold with metric $g$ and three complex structures $I, J$ and $K$ satisfying three conditions:

1. $I J=-I J=K$ so $I, J, K$ form an algebra of quaternions.

2. $I, J$ and $K$ are covariantly constant with respect to $g$.

3. $g$ is Hermitian with respect to all of the complex structures.

From these conditions, it follows that there are three Kähler forms associated to each complex structure, $\omega_{1}, \omega_{2}$ and $\omega_{3}$. Suppose $X$ is a hyperKähler manifold admitting an action of the Lie group $G$ preserving the metric and the three complex structures. For the case of gauge theories, $X$ is the space of hypermultiplet VEVs. The group $G$ preserves the three Kähler forms, and any one of the Kähler forms $\omega_{i}$ defines a symplectic structure on $X$. Therefore the action of $G$ is generated by a Hamiltonian $\mu_{i}$ valued in $\mathbf{g}^{*}$, the dual of the Lie algebra of $G$. Together these three Hamiltonians define a moment map $\mu$ from $X$ to $\mathbf{g}^{*} \otimes \mathbb{R}^{3}$. It follows from these definitions that the level set of $\mu, \mu^{-1}(0)$, is invariant with respect to $G$. Then according to the theorem of [16], the quotient $\mu^{-1}(0) / G$ is a hyperKähler space.

Let us consider a particular example of hyperKähler quotient. Let the group $U(N)$ act by left multiplication on the flat hyperKähler manifold $\mathbb{H}^{N}$. 
This action commutes with right multiplication by $I, J, K$ and therefore preserves all three complex structures. The corresponding moment map is most compactly written if we think of $\mathbb{H}^{N}$ as $\mathbb{C}^{N} \times \mathbb{C}^{N}$ with coordinates $(Q, \tilde{Q})$. Then the moment map is given by

$$
\mu_{1}=i\left(Q \otimes Q^{\dagger}-\tilde{Q}^{\dagger} \otimes \tilde{Q}\right), \quad \mu_{2}-i \mu_{3}=2 i Q \otimes \tilde{Q} .
$$

In this particular case, the hyperKähler quotient $\mu^{-1}(0) / G$ is a single point $Q=\tilde{Q}=0$.

In the case of impurity theories, we have seen that the Higgs branch is described by the moduli spaces of solutions of differential rather than algebraic equations. In the cases that we considered, these equations are $\mathrm{Nahm}$ or Hitchin equations with point-like impurities. As expected on general grounds, these moduli spaces are hyperKähler spaces. A way to see this is to note that these equations can be regarded as moment map equations for an infinite-dimensional group of gauge transformations $\mathcal{G}$ [17]. For the case considered in section 2.2, where space was a circle, we obtained Nahm equations. The corresponding infinite-dimensional gauge group $\mathcal{G}$ is the loop group of $G$, i.e. it is the group of maps from $\widehat{S}^{1}$ to $G$. This group acts on an infinite-dimensional flat hyperKähler manifold consisting of all quadruplets $\left(T_{0}, T_{1}, T_{2}, T_{3}\right)$, where $T_{0}$ is a $U(N)$ connection on $\widehat{S}^{1}$ and $T_{i}$ are adjointvalued fields on $\widehat{S}^{1}$. The model in section 2.3 lived on $\widehat{T}^{2}$, and its Higgs branch was described by Hitchin equations. In this case, $\mathcal{G}$ is the group of maps from $\widehat{T}^{2}$ to $G$. It acts on the space of quadruplets $\left(A_{z}, A_{\bar{z}}, \Phi, \Phi^{\dagger}\right)$, where $A_{z} d z+A_{\bar{z}} d \bar{z}$ is a $U(N)$ connection on $\widehat{T}^{2}$ and $\Phi d z+\Phi^{\dagger} d \bar{z}$ is an adjointvalued 1-form on $T^{2}$. In both cases the theorem of [16] guarantees that the space of solutions modulo gauge transformations is a hyperKähler space.

To account for the impurity degrees of freedom, we have to enlarge the initial configuration space by including the fundamental hypermultiplets living at $k$ points $z_{1}, \ldots, z_{k}$ on either $\widehat{S}^{1}$ or $\widehat{T}^{2}$. Each fundamental hypermultiplet takes values in $\mathbb{H}^{N}$, and we have just seen that there is an action of $G=U(N)$ on $\mathbb{H}^{N}$ preserving the hyperKähler structure. Thus there is a natural action of $\mathcal{G}$ on the enlarged space of variables, with $g(z) \in \mathcal{G}$ acting on the $p^{\text {th }}$ hypermultiplet $Q_{p}$ by $Q_{p} \rightarrow g\left(z_{p}\right) Q_{p}$. The moment map then contains an extra contribution proportional to a sum of delta functions. Let us denote the moment map for the bulk variables by $\mu_{\mathcal{G}}$, and the moment map for the degrees of freedom localized at $z_{p}$ by $\rho_{p}$. The explicit form of $\rho_{p}$ for the action of $U(N)$ on $\mathbb{H}^{N}$ is given in eq. (3.1). Then the combined moment map $\mu_{T}$ is given by,

$$
\mu_{T}=\mu_{\mathcal{G}}+\sum_{p} \delta\left(z-z_{p}\right) \rho_{p}
$$

The equations (2.11) and (2.13) for the supersymmetric vacua of our impurity models are exactly of this form. 


\subsection{The Relation to Solutions of Self-duality Equations}

The impurity model discussed in section $2.2(N$ D0-branes bound to $k$ parallel D4-branes compactified on a circle) describes $N U(k)$ instantons on $\mathbb{R}^{3} \times S^{1}$. Instantons on $\mathbb{R}^{3} \times S^{1}$ are also known as calorons [18]. Thus we infer that the moduli space of calorons coincides with the moduli space of Nahm equations on a circle with point-like impurities. In this case, we have obtained a known result which can be derived in several different ways $[18,19]$. The novelty in our derivation is that we obtain the answer by studying the dynamics of an impurity system. This kind of approach was first used in [20] where type I 5-branes in flat space were studied. In that case, the gauge theory was an ordinary gauge theory without impurities, and so the equation $\mathcal{D}=0$ was algebraic. The $\mathcal{D}$-flatness conditions were identical to the equations arising in the ADHM construction of instantons on $\mathbb{R}^{4}$. This agreed with the expectation that the Higgs branch of type I 5-branes corresponds to finite size instantons in the transverse $\mathbb{R}^{4}$.

In the case considered in section 2.3 (D0-branes bound to D4-branes compactified on $T^{2}$ ) we obtained Hitchin equations rather than Nahm equations. We are going to argue that the moduli space of Hitchin equations on the dual torus $\widehat{T}^{2}$ with extra degrees of freedom in the fundamental, localized at punctures, correctly describes the moduli space of $U(k)$ instantons on $\mathbb{R}^{2} \times T^{2}$. It seems that instantons on $\mathbb{R}^{2} \times T^{2}$ have not been discussed in the literature, so in this section we intend to fill this gap. But before doing so, we recall how Nahm equations come about in the case of calorons.

The correspondence between solutions of self-duality equations on $\mathbb{R}^{3} \times S^{1}$ and Nahm equations on $\widehat{S}^{1}$ is known as the Nahm transform [18, 21]. The $\mathrm{Nahm}$ transform maps every solution of the former into a solution of the latter in the following way. Consider a self-dual $U(k)$ connection $A$ on $\mathbb{R}^{3} \times S^{1}$ with an asymptotic Wilson line at spatial infinity breaking $U(k)$ down to $U(1)^{k}$. The topological invariants characterizing such a connection are its second Chern class $N$ and its $k-1$ magnetic charges with respect to the unbroken $U(1)$ factors; the charge in the diagonal $U(1)$ subgroup is always zero. We consider normalizable solutions of the Dirac equation,

$$
\sigma \cdot D_{A} \psi(x, s)=i s \psi(x, s)
$$

where $\psi$ is in the fundamental representation of $U(k)$ and $s$ is a real parameter. Because $e^{i s}$ can be regarded as an auxiliary $U(1)$ Wilson line around $S^{1}, s$ is a periodic variable living on the dual circle $\widehat{S}^{1}$. The number of normalizable zero modes $\psi(x, s)$ is locally constant in $s$, but may have jumps at points $e^{i s}=W_{p}$ where $W_{p}, p=1, \ldots, k$, are the eigenvalues of the $U(k)$ 
Wilson line at spatial infinity. We next define four $s$-dependent matrices:

$$
\begin{aligned}
& T_{i}(s)=i \int \psi^{\dagger} x_{i} \psi d^{4} x, i=1,2,3 \\
& T_{0}(s)=\int \psi^{\dagger} \frac{\partial}{\partial s} \psi d^{4} x .
\end{aligned}
$$

The dimension of $T_{i}, T_{0}$ is equal to the number of zero modes $\psi(x, s)$ and therefore may also jump at $e^{i s}=W_{p}$. Away from the jumping points, $T_{i}(s), T_{0}(s)$ satisfy Nahm equations [18]. The behavior near the jumping points is generally complicated [14], but simplifies when the dimension of $T_{i}, T_{0}$ is independent of $s$ and equal to $N$, the second Chern class of the gauge connection. This happens when the original gauge connection has no magnetic charges, and corresponds to the brane configuration considered in section 2.2. In this special case, the matrices $T_{i}, T_{0}$ have finite left and right limits near $s=s_{p} \equiv-i \log W_{p}, p=1, \ldots, k$ and satisfy

$$
T_{0}\left(s_{p}+0\right)=T_{0}\left(s_{p}-0\right), \quad T_{i}\left(s_{p}+0\right)-T_{i}\left(s_{p}-0\right)=\rho_{i}\left(Q_{p}\right) .
$$

Here $Q_{p}, p=1, \ldots, k$, are elements of $\mathbb{H}^{N}$, and $\rho(Q)$ is the moment map for the natural action of $U(N)$ on $\mathbb{H}^{N}$ given in eq. (3.1). These conditions can be combined with the Nahm equations into,

$$
\frac{d T_{i}}{d s}+\left[T_{0}, T_{i}\right]+\frac{1}{2} \epsilon_{i j k}\left[T_{j}, T_{k}\right]=\sum_{p} \rho_{i}\left(Q_{p}\right) \delta\left(s-s_{p}\right) .
$$

The latter equation has the form eq. (3.2) and coincides with the condition of $\mathcal{D}$-flatness in section 2.2 , as claimed.

Conversely, given a solution of Nahm equations one can reconstruct the gauge connection $A$. We are not going to describe the inverse Nahm transform here, and simply remark that it can be obtained by studying the dynamics of a probe brane along the lines discussed in [4, 22].

We would like to stress that the correct jumping conditions are absolutely crucial in order to get the right moduli space. In our discussion they came out naturally from the analysis of the strings stretched between D0 and D4branes. An attentive reader has probably noticed a close connection between the brane configuration of section 2.2 and that considered in [22]. We want to point out an important difference between the two. When we perform a T-duality on the D4-D0 configuration of section 2.2 along the compact direction, we obtain a system of $N$ parallel D1-branes compactified on a circle with impurities representing the $k$ D3-branes. On the other hand, the system studied in [22] contained $N$ D1-branes suspended between two parallel D3-branes. The suspended D1-brane corresponds to a monopole in the world-volume theory of the D3-branes. The moduli space of monopoles is 
described by Nahm equations on an interval $s \in(0,1)$ where the $T_{i}$ matrices have poles at the ends of the interval $[18,17]$. It was shown in [22] that Nahm equations indeed follow naturally from an analysis of the dynamics of D1branes, with $s$ parallel to the world-volume of the D1-branes. However in the case of suspended $D 1$-branes, it seems difficult to derive the right boundary conditions for the $T_{i}$ matrices from considerations of brane dynamics. In principle, the correct boundary conditions should be derivable from our setup by taking a limit where some of suspended D1-brane segments are taken to infinity in the $x^{2}, x^{3}, x^{4}$ directions.

We now turn to the less familiar and more interesting case of $U(k)$ instantons on $\mathbb{R}^{2} \times T^{2}$. By an instanton, we mean a connection $A$ with self-dual curvature $F_{A}$ and finite action. In particular, this means that the second Chern class is well-defined. We allow for non-trivial Wilson lines at infinity breaking $U(k)$ down to $U(1)^{k}$. The possible Wilson lines are parametrized by $k$ unordered points $z_{1}, \ldots, z_{k}$ on the dual torus $\widehat{T}^{2}$. No magnetic charges are allowed because with only two noncompact dimensions, the magnetic field of a monopole would decay as $1 / r$ and the action would diverge logarithmically. Let us denote the holomorphic coordinate on $\mathbb{R}^{2}$ by $t$ and the holomorphic coordinate on $T^{2}$ by $w$. To perform the Nahm transform, we again look for solutions of the Dirac equation with auxiliary $U(1)$ Wilson lines. These auxiliary parameters live on the dual torus $\widehat{T}^{2}$ whose complex coordinate we call $z$. For generic values of $z$, the Dirac equation will have $N$ normalizable zero modes (we assume here that the connection $A$ on $\mathbb{R}^{2} \times T^{2}$ can be extended to a connection on the compactified space $S^{2} \times T^{2}$ ). In fact, we expect these zero modes to decay exponentially at infinity. Then we can define Nahm matrices living on $\widehat{T}^{2}$,

$$
A_{z}=\int \psi^{\dagger} \frac{\partial}{\partial z} \psi d^{2} t d^{2} w, \quad \Phi=i \int \psi^{\dagger} t \psi d^{2} t d^{2} w .
$$

Using arguments along the lines of $[18,23]$, it can be shown that $A_{z}$ and $\Phi$ satisfy Hitchin equations away from $z=z_{p}, p=1, \ldots, k$. As $z \rightarrow z_{p}$ the norm of some zero modes of the Dirac operator diverges, and therefore $A_{z}$ and $\Phi$ diverge as well. We therefore expect that $A_{z}$ and $\Phi$ will have poles at $z=z_{p}, p=1, \ldots, k$.

The argument of the preceeding paragraph shows that the Nahm transform of a self-dual $U(k)$ connection $A$ on $\mathbb{R}^{2} \times T^{2}$ with second Chern class $N$ is a solution of the $U(N)$ Hitchin equations on the dual torus $\widehat{T}^{2}$ with poles at $z=z_{p}, p=1, \ldots, k$. The position of the punctures encodes the asymptotic eigenvalues of the $U(k)$ Wilson lines. This is in complete agreement with eq. (2.13) obtained from studying brane dynamics. We have not established the precise kind of the singularities that $A_{z}$ and $\Phi$ should have, but we believe that we have presented enough evidence that eq. (2.13) correctly describes the moduli space of instantons on $\mathbb{R}^{2} \times T^{2}$. Granted this, 
eq. (2.13) tells us what kind of singularities the Nahm matrices must have. For example, $\Phi$ has a pole at $z=z_{p}$ with residue proportional to $Q^{p} \otimes \tilde{Q}^{p}$. It remains an interesting challenge to derive this result using either the Nahm transform [21] or twistor methods [17].

\subsection{Some Examples of Moduli Spaces}

Let us discuss some simple examples of instanton moduli spaces on $\mathbb{R}^{3} \times S^{1}$ and $\mathbb{R}^{2} \times T^{2}$. We remind the reader that we are only discussing instanton configurations with no magnetic charges.

First we give examples of moduli spaces of instantons on $\mathbb{R}^{3} \times S^{1}$. Some interesting recent discussion of these instanton solutions appeared in [24]. For $N=1$, the Nahm equations require that $T_{i}$ be locally constant with discontinuities occuring at $s=s_{p}, p=1, \ldots, k$. The center of mass degrees of freedom clearly live in $\mathbb{R}^{3} \times S^{1}$, while the rest of the moduli space can be shown to be a $4 k-4$-dimensional manifold endowed with a 'periodic' version of the Lee-Weinberg-Yi metric $[25,26]$. It has $k-1$ compact directions and a singularity at the origin. In particular, for $k=2$ it reduces to a $\mathbb{Z}_{2}$ orbifold of the Taub-NUT metric. It is also interesting to look at the limit when $k^{\prime} \leq k$ of the points $z_{p}$ coincide. This is also the limit in which the broken gauge group $U(k)$ is partially restored to $U\left(k^{\prime}\right)$. In this limit, $k^{\prime}-1$ compact directions of the moduli space decompactify but the moduli space description seems to remain valid [25]. Note that turning on the FI deformation will smooth out the singularity of the moduli space.

For $N=2$, the Nahm equations can be solved in terms of elliptic functions; see [27] for example. The resulting moduli space splits into a product of $\mathbb{R}^{3} \times S^{1}$ and a fairly complicated $8 k-4$-dimensional hyperKähler manifold. We have not investigated the detailed properties of this manifold; we simply remark that it has $2 k-1$ compact directions. In this case, it seems that the limit where $U\left(k^{\prime}\right)$ symmetry is restored does not lead to decompactification of the moduli space describing the relative motion of the D1-branes. This is in accord with our intuition from the matrix model interpretation, which we will discuss in section four. In general, it is easy to see that the moduli space of $N U(k)$ calorons with no magnetic charges has dimension $4 N k$ and a total of $N k$ compact directions.

Now let us turn to instantons on $\mathbb{R}^{2} \times T^{2}$. Here we only discuss the case $N=1$. The situation is quite different from the case of instantons on $\mathbb{R}^{3} \times S^{1}$. The solutions of the Hitchin equations with impurities depend on $4 k$ parameters. However, not all of the parameters correspond to normalizable zero modes. The mathematical reason goes as follows: the Hitchin equations are abelian in this case and easily solved in terms of elliptic functions with simple poles at $z=z_{p}$ for $p=1, \ldots, k$. The residues of these elliptic functions are determined by the variables $Q$ and $\tilde{Q}$. Therefore, the 
tangent vectors associated to varying $Q$ and $\tilde{Q}$ also have simple poles. The norm of these tangent vectors is then logarithmically divergent. In this case where $N=1$, the only finite norm tangent vector corresponds to a constant solution. It is then easy to see that the moduli space is just $\mathbb{R}^{2} \times T^{2}$.

In the limit where two or more impurities collide, so some non-abelian gauge symmetry is restored, the dimension of the moduli space becomes larger. This can be seen by noting that even if we require the residue at the collision point to remain fixed, $Q$ and $\tilde{Q}$ have a non-trivial moduli space when $k>1$. This moduli space is the same as the Higgs branch of a $U(1)$ gauge theory with $k$ electrons.

For $N>1$, the story is similar: $4 k-4$ zero modes freeze out and the dimension of the resulting moduli space is $4(N k-k+1)[3,28]$. When some impurities collide, the dimension of the moduli space will again jump. It would be very interesting to analyze the structure of the moduli space for $N>1$ in detail.

\section{Some Applications of Impurity Models}

\subsection{Matrix Models for $S U(k)$ Yang-Mills Theories}

A natural way to construct $\mathrm{N}=4 U(k)$ Yang-Mills is to compactify $k$ parallel $\mathrm{M}$ theory five-branes on $T^{2}$. The $U(1)$ describing the center of mass motion effectively decouples, leaving an $S U(k)$ theory. At energies well below the eleven-dimensional Planck mass $M_{p l}$ and the scale set by the torus, the theory reduces to Yang-Mills in four dimensions. The complex structure of the torus determines the coupling of the Yang-Mills theory, and S-duality is therefore made manifest [29]. In this section, we will describe the DLCQ description of $k$ parallel M theory five-branes wrapped on either $S^{1}$ or $T^{2}$. The DLCQ description involves the Higgs branches of the impurity models that we have considered [3].

Longitudinal five-branes are described in matrix theory by D4-branes [30]. To obtain a matrix formulation of $k$ compactified five-branes in the sector with $P^{+}=N / R_{\|}$, we need to consider the dynamics of $N$ D0-branes in the presence of $k$ wrapped D4-branes. We will follow the usual prescription [31]. Longitudinal five-branes wrapped on $S^{1}$ or $T^{2}$ give longitudinal D4 and D3-branes, respectively. Therefore, longitudinal D4 or D3-branes are represented by impurities in the $1+1$ and $2+1$-dimensional field theories describing D0-branes on $S^{1}$ and $T^{2}$. The spacetime transverse to the longitudinal branes is described by the Coulomb branch of the impurity theories, where $Y$ has non-zero expectation value. The compactified $(2,0)$ theory in DLCQ is then described by the other branch of the impurity theory - the Higgs branch. This is analogous to the uncompactified $(2,0)$ case $[32,33]$. 
Note that if we turn on the FI deformation, the Coulomb branch is lifted and so the Higgs branch decouples from spacetime.

Let us consider the more interesting case of $S U(k) \mathrm{N}=4$ Yang-Mills on parallel longitudinal D3-branes. Let $R_{1}, R_{2}$ be the radii of the torus $T^{2}$ on which we compactify the five-branes. For simplicity, let us assume the torus is rectangular. At energies $E<<1 / R_{1}, 1 / R_{2}$, the effective theory on the compactified five-branes is Yang-Mills. We hold fixed the Yang-Mills coupling,

$$
g_{Y M}^{2}=\frac{R_{1}}{R_{2}}
$$

which is the same as the type IIB string coupling. When the area of the torus is finite, we are describing the type IIB string in DLCQ with transverse space $\mathbb{R}^{7} \times S^{1}$, where the circle has size $\frac{1}{M_{s}^{2} R_{2}}$. The longitudinal D3-branes are transverse to the compact circle. The string scale is given in terms of the eleven-dimensional Planck scale: $M_{s}^{2}=M_{p l}^{3} R_{1}$.

The matrix model for this system is the 2+1-dimensional impurity model of section 2.3 with coupling constant,

$$
g_{m a t}^{2}=\frac{R_{\|}}{R_{1} R_{2}}
$$

The theory lives on the dual torus with sides $\Sigma_{1}, \Sigma_{2}$ where,

$$
\Sigma_{i}=\frac{1}{M_{p l}^{3} R_{\|} R_{i}}
$$

Since we are considering energies far below $M_{s}$, we can ignore modes with a non-trivial dependence on the two spatial directions of this dual torus. Our $2+1$-dimensional theory then reduces to quantum mechanics on the Higgs branch moduli space. Note that S-duality is manifest in this formulation. The coupling constant in the quantum mechanical sigma model diverges,

$$
g_{Q M}^{2}=\frac{g_{m a t}^{2}}{\Sigma_{1} \Sigma_{2}} \rightarrow \infty
$$

so we are left with a conformal theory on the Higgs branch. There are compact directions on the moduli space, but the energies associated to excitations along those directions are much higher than the energies we are considering [3]. We can therefore take wavefunctions which are slowly varying along the compact directions. This is the matrix model analogue of ignoring Kaluza-Klein modes along $T^{2}$.

The case when the impurities are separated on the dual torus corresponds to Yang-Mills with light-like Wilson lines. These light-like Wilson lines are 
determined by points on $T^{2}$ rather than $S^{1}$ because four-dimensional YangMills in light-cone is effectively three-dimensional. Therefore the compact scalars dual to the three-dimensional gauge fields can also be given expectation values. Note that as $N \rightarrow \infty$, the light-like Wilson lines should disappear and we should recover a description of the interacting conformal field theory.

We noted in the previous section that certain zero modes present in the $S^{1}$ case are frozen out in the $T^{2}$ case. Recall that some zero modes seem to be restored when two or more impurities collide. This corresponds to the limit where some non-abelian gauge symmetry is restored. We should then optimistically be describing the interacting conformal field theory on coincident D3-branes in DLCQ at finite $N$. If we turn on a FI deformation, the moduli space is smooth, even in the case of coincident impurities. It is important to determine whether this matrix description actually captures all the degrees of freedom needed to describe the conformal field theory at finite $N$. This depends largely on how much can be understood about the moduli space of instantons on $\mathbb{R}^{2} \times T^{2}$ with no Wilson lines, or perhaps about its non-commutative generalization. Similarly, the matrix description of $M$ theory five-branes wrapped on $S^{1}$ is given by the impurity theory considered in section 2.2 .

\subsection{Non-commutative Instantons and Coulomb Branches}

Impurity theories can be used to solve for the Coulomb branches of certain conventional $\mathrm{N}=2 \mathrm{~d}=4$ gauge theories. For the specific model studied in section 2.3, the Higgs branch is related to the Coulomb branch of the four-dimensional theory obtained by placing $N$ D3-branes on an $A_{k-1}$ singularity [3]. This quiver theory has gauge group $U(N)^{k}$ with a chain of bifundamentals [34]. All the $U(1)$ factors except for the diagonal $U(1)$ factor will become free in the infra-red. This is the Coulomb branch analogue of the freezing of moduli noted in section 3.3. There is a T-dual realization of this configuration in terms of $N$ D4-branes wrapped on a compact circle with $k$ NS five-branes located at points on the circle [35]. This configuration is T-dual to an $A_{k-1}$ singularity when the NS five-branes are coincident.

We have the freedom of turning on a FI parameter in our impurity model, which lifts the Coulomb branch and deforms the Higgs branch. The Higgs branch can then be interpreted as describing instantons on a noncommutative $\mathbb{R}^{2} \times T^{2}$. How does this deformation appear in the quiver theory and its T-dual realization? The quiver theory has a single mass parameter. Turning on the mass lifts the Higgs branch, which in the matrix model interpretation describes motion in the transverse spacetime. This deformation corresponds to turning on the FI parameter in the impurity model. In the T-dual description with D4-branes and NS five-branes, it corresponds to placing the branes in a non-trivial background [35]. 
Let us discuss in some detail the case of $\mathrm{N}=2 U(N)$ gauge theory with a massive adjoint hypermultiplet. In discussing the relevant brane configurations, we will follow the conventions of [35]. The brane configuration contains $N$ D4-branes wrapped on a circle with a single NS five-brane located at a point on the circle parametrized by $x^{6}$. The motion of the D4-branes in the directions parallel to the NS five-brane is parametrized by a complex coordinate $v=x^{4}+i x^{5}$. In the case without the mass deformation, we consider $\mathrm{M}$ theory on $T^{2}$ with some five-branes. The circle on which we reduce $\mathrm{M}$ theory to type IIA is parametrized by $x^{10}$. To turn on the mass deformation, we take an affine $\mathbb{C}$-bundle over the torus parametrized by $x^{6}$ and $x^{10}$. The fiber $\mathbb{C}$ is coordinatized by $v$. As we go around the circle parametrized by $x^{6}, v$ is shifted by a complex number $m$. In this case, the low-energy theory on the D4-branes is $U(N)$ Yang-Mills with an adjoint hypermultiplet with mass $m$. The Coulomb branch was determined in [36, 35]. Using the Higgs branch of impurity theories, we can determine this Coulomb branch in a quite different way.

We consider the theory of section 2.2 with a single D4 wrapped on $T^{2}$ and $N$ D0-branes. If we do not turn on the FI deformation, the Higgs branch is quite trivial. It is $S^{N}\left(\mathbb{R}^{2} \times T^{2}\right)$, and this agrees with the Seiberg-Witten fibration which describes the Coulomb branch of $N=4 U(N)$ Yang-Mills. The complex structure of the $T^{2}$ is determined by the coupling of the YangMills theory. If we turn on the FI deformation, the Higgs branch becomes non-trivial and is given by the moduli space of solutions of eq. (2.13) with $k=1$,

$$
\begin{gathered}
F_{z \bar{z}}-\left[\Phi, \Phi^{\dagger}\right]=\frac{1}{2 R_{1} R_{2}} \delta^{2}(z)\left(Q \otimes Q^{\dagger}-\tilde{Q}^{\dagger} \otimes \tilde{Q}\right), \\
\bar{D} \Phi=-\frac{1}{2 R_{1} R_{2}} \delta^{2}(z)(Q \otimes \tilde{Q}-\xi) .
\end{gathered}
$$

The FI deformation $\xi$ of the impurity theory is proportional to the mass deformation $m$ of the Coulomb branch. To compare with the solution of [36], let us consider eq. (4.4) modulo the action of the complexified gauge group. This is sufficient to determine the complex structure of the Seiberg-Witten fibration. First consider the trace of eq. (4.4). This gives,

$$
\bar{\partial} \operatorname{Tr} \Phi=-\frac{1}{2 R_{1} R_{2}} \delta^{2}(z)(\tilde{Q} Q-N \xi) .
$$

This implies that $\operatorname{Tr} \Phi$ is a meromorphic function on $T^{2}$ with a single pole at $z=0$. The residue must therefore be zero, forcing:

$$
\tilde{Q} Q=N \xi \text {. }
$$


The system described by eq. (4.4) and eq. (4.6) has been considered in [37], and shown to be equivalent to the (complexified) elliptic Calogero-Moser system. In turn, the Calogero-Moser system is equivalent to the DonagiWitten solution [38]. Concretely, this means the following: the CalogeroMoser system is integrable, so it has $N$ Poisson commuting integrals of motion. There are $N$ pairs of action-angle variables, and the phase space is therefore fibered by tori of angle variables. After complexification, this fibration becomes equivalent to the Seiberg-Witten fibration describing softly broken $N=4 U(N)$ Yang-Mills. This approach to solving $N=2 \mathrm{~d}=4$ gauge theories will be further explored in [28].

\section{Acknowledgments}

It is our pleasure to thank S. Cherkis, O. Ganor, G. Moore, N. Nekrasov, J. Park, A. Uranga, C. Vafa and E. Witten for helpful discussions. The work of A.K. is supported by DOE DE-FG02-90ER40542; that of S.S. by NSF grant DMS-9627351.

\section{References}

[1] T. Banks, W. Fischler, S. H. Shenker, and L. Susskind, Phys. Rev. D55 (1997) 5112.

[2] S. Sethi, hep-th/9710005.

[3] O. Ganor and S. Sethi, J. High Energy Phys. 1 (1998) 007.

[4] M. Douglas, hep-th/9512077; hep-th/9604198.

[5] A. Gerasimov, G. Moore, and S. Shatashvili, unpublished.

[6] D. Tsimpis, to appear.

[7] W. Taylor IV, Phys. Lett. B394 (1997) 283; hep-th/9801182.

[8] O. Ganor, S. Ramgoolam, and W. Taylor IV, Nucl. Phys. B492 (1997) 191.

[9] P. West, "Introduction to Supersymmetry and Supergravity", World Scientific 1990.

[10] P. Horava and E. Witten, Nucl. Phys. B475 (1996) 94.

[11] M. Berkooz, hep-th/9802069; O. Aharony, M. Berkooz, and N. Seiberg, hep-th/9712117. 
[12] A. Connes, M. Douglas, and A. Schwarz, hep-th/9711162; M. Douglas and C. Hull, hep-th/9711165.

[13] N. Nekrasov and A. Schwarz, hep-th/9802068.

[14] J. Hurtubise and M.K. Murray, Comm. Math. Phys. 122 (1989) 35.

[15] C. Ahn and B.-H. Lee, hep-th/9803069.

[16] N.J. Hitchin, A. Karlhede, U. Lindström, and M. Roček, Comm. Math. Phys. 108 (1987) 535.

[17] N.J. Hitchin, Comm. Math. Phys. 83 (1982) 579.

[18] W. Nahm, "Self-Dual Monopoles and Calorons", in "Lect. Notes in Phys. 201", Springer 1984.

[19] H. Garland and M.K. Murray, Comm. Math. Phys. 120 (1988) 335.

[20] E. Witten, Nucl. Phys. B460 (1996) 541.

[21] W. Nahm, "The Construction of All Self-Dual Monopoles by the ADHM Method,", in "Monopoles in Quantum Field Theory", eds. N. S. Craigie et al., World Scientific 1982.

[22] D.-E. Diaconescu, Nucl. Phys. B503 (1997) 220.

[23] E. Corrigan and P. Goddard, Ann. Phys. 154 (1984) 253.

[24] K. Lee, hep-th/9802012; K. Lee and C. Lu, hep-th/9802108; T. C. Kraan and P. van Baal, hep-th/9802049.

[25] K. Lee, E. Weinberg, and P. Yi, Phys. Rev. D54 (1996) 1636.

[26] K. Lee and P. Yi, Phys. Rev. D56 (1997) 3711.

[27] A.S. Dancer, Comm. Math. Phys. 158 (1993) 545.

[28] A. Kapustin, to appear.

[29] E. Witten, hep-th/9507121.

[30] M. Berkooz and M. Douglas, Phys. Lett. B395 (1997) 196.

[31] N. Seiberg, Phys. Rev. Lett.79 (1997) 3577; A. Sen, hep-th/9709220.

[32] O. Aharony, M. Berkooz, S. Kachru, N. Seiberg, and E. Silverstein, Adv. Theor. Math. Phys. 1 (1998) 148.

[33] E. Witten, J. High Energy Phys. 7 (1997) 003. 
[34] M. Douglas and G. Moore, hep-th/9603167.

[35] E. Witten, Nucl. Phys. B500 (1997) 3.

[36] R. Donagi and E. Witten, Nucl. Phys. B460 (1996) 299.

[37] A. Gorsky and N. Nekrasov, hep-th/9401021.

[38] H. Itoyama and A. Morozov, Nucl. Phys. B477 (1996) 855. 